\title{
Editorial
}

\section{Liebe Leserinnen und Leser}

Die Transformation vom Produkt- zum Dienstleistungsunternehmen ist in den letzten Jahren zunehmend in den Mittelpunkt des Interesses von Wissenschaft und Praxis gerückt. Insbesondere in den Industriegüterbranchen haben die steigende Wettbewerbsintensität, ein damit einhergehender Preisdruck, die zunehmende Angleichung von Produktangeboten dazu geführt, dass Unternehmen nicht mehr nur noch reine Produktangebote gestalten und vermarkten, sondern - im Rahmen der Transformation zum Dienstleistungsunternehmen zunehmend Dienstleistungen in das Portfolio aufnehmen. Hierdurch ergeben sich unter anderem Differenzierungschancen, die Möglichkeit zum Aufbau enger Kundenbeziehungen und insbesondere auch ökonomische Potenziale. Oft verändern sich mit der Transformation auch die Geschäftsmodelle und es kommt zu einer Verlagerung der Wertschöpfung von einer Produktwertschöpfung hin zu einer Servicewertschöpfung.

Für die Unternehmenspraxis lässt sich zum aktuellen Zeitpunkt feststellen, dass der Entwicklungsstand im Industriegütersektor hinsichtlich der Transformation vom Produktzum Dienstleistungsunternehmen nicht weit vorangeschritten ist. Des weiteren zeigt sich, dass es zahlreiche Umsetzungsbarrieren bei der Servicetransformation in Unternehmen gibt, die vielfach zum Scheitern der Servicetransformation führen.

Vor dem Hintergrund der zunehmenden Bedeutung und der zahlreichen damit verbundenen Problemstellungen hat sich die Transformation vom Produzenten zum Dienstleister zu einem prominenten Forschungsthema entwickelt, was sich unter anderem in der kontinuierlich steigenden Anzahl an wissenschaftlichen Artikeln widerspiegelt. Mit der Transformation vom Produkt- zum Dienstleistungsunternehmen werden Fragestellungen von der Entwicklung von innovativen Dienstleistungsangeboten, der Finanzierung, dem Marketing bis hin zu der Organisation und Führung des sich zum Dienstleistungsanbieter entwickelnden Produktunternehmens aufgeworfen.

Das vorliegende Themenheft „Transformation vom Produkt- zum Serviceunternehmen“ widmet sich einigen dieser Fragestellungen. Bei der Konzeption des Heftes sowie der Beitragsauswahl wurde sowohl der theoretische Anspruch als auch die praktische Relevanz berücksichtigt. Damit wendet sich das Heft sowohl an Wissenschaftler, Studierende als auch an Praktiker.

Einen Blick auf die aktuelle und zukünftige Entwicklung der Forschung zur Transformation vom Produkt- zum Serviceunternehmen werfen die Autoren Heiko Gebauer und Caroline Saul. Sie führen in ihrem Beitrag eine bibliometrische Analyse des Forschungsstandes und der zukünftigen Forschungsfragen für die Transformation vom Produzenten zum Dienstleister durch. Neben der Differenzierung verschiedener Entwicklungsphasen der Servicetransformations-Forschung seit Ende der 1980er Jahre werden mittels Zitationsanalyse und Faktorenanalyse sieben Forschungsrichtungen identifiziert: Erbringung von Lösungen, Vermarktung von Lösungen, Dienstleistungen in produzierenden Unternehmen, Erfolg im Dienstleistungsgeschäft, Produkt-Service-Systeme und Dienstleistungsentwicklung. Aufbauend auf dieser Analyse formulieren die Autoren empirische und theoretische Handlungsempfehlungen für die zukünftige Ausgestaltung des Forschungsthemas. 
Die Rolle der Digitalisierung bei der Transformation vom Produzenten zum produzierenden Dienstleister analysieren Christian Lerch und Matthias Gotsch in ihrem Beitrag und richten damit einen Blick auf eine sehr aktuelle Entwicklung in vielen Industriegüterbranchen. Klassische Modelle für industrielle Dienstleistungen sind zwar in der Lage die zunehmende Tertiarisierung vieler Industriegüterbranchen zu erklären, vernachlässigen aber gleichzeitig die zunehmende Digitalisierung vieler industriell angebotener Dienstleistungen. Durch die Kombination der beiden Entwicklungen - Tertiarisierung und Digitalisierung - ergeben sich zahlreiche Auswirkungen auf die Transformation vom Produzenten zum produzierenden Dienstleister. Vor diesem Hintergrund entwickeln die beiden Autoren ein neues Transformationsmodell, das unter Berücksichtigung der Tertiarisierung und Digitalisierung ein neues Bezugssystem für das Dienstleistungsangebot von Produktherstellern umfasst.

Bisherige Forschungsarbeiten betrachten die Transformation vom Produkt- zum Dienstleistungsunternehmen nahezu ausschließlich aus der Anbietersicht. Dabei stellt die Voraussetzung einer erfolgreichen Transformation auch deren kundenseitige Akzeptanz dar. Vor diesem Hintergrund analysieren Jens Hogreve und Richard Wonner das implizite Wissen als Determinante des Kaufverhaltens produktbegleitender Dienstleistungen auf Industriegütermärkten. Der Beitrag führt hierzu eine explorative Analyse auf Basis von qualitativen Kundeninterviews durch. Die Ergebnisse zeigen, dass das Kaufverhalten stark von Situation und Unternehmenskultur des Kunden geprägt wird. Dabei nimmt implizites Wissen eine Schlüsselrolle ein, da es den Wissensvorsprung des Anbieters schmälert und Kunden veranlasst, eigenes Personal zur Leistungserbringung aufzubauen.

In meinem gemeinsam mit Henrik Vogel verfassten Beitrag wird mit der Systemlösungsorientierung eine Strategieoption der Servicetransformation betrachtet. Es werden die Einflussfaktoren auf den Umsetzungserfolg einer Systemlösungsstrategie im Kontext des Vertriebs analysiert. Im Kern geht es um die Fragestellung der Gestaltung von institutionellen und personellen Rahmenbedingungen, damit die Transformation von einem Produkthersteller zu einem Serviceunternehmen gelingt. Im Rahmen einer kausalanalytischen Untersuchung werden die Einflussstärken zentraler Unternehmensparameter auf die systemlösungsorientierte Vertriebseinstellung von Verkaufsmitarbeitern ermittelt. Die Ergebnisse belegen einen besonders starken Einfluss der Unternehmenskultur und der Anreizgestaltung. Des Weiteren wird die systemlösungsorientierte Vertriebseinstellung als Treiber des individuellen Verkaufserfolges mit Systemlösungen nachgewiesen.

Ich wünsche Ihnen eine anregende Lektüre und hoffe, dass das Themenheft „Transformation vom Produkt- zum Serviceunternehmen“ nicht nur der Forschung neue Impulse gibt, sondern auch zu einem verstärkten Theorie-Praxis-Dialog anregt.

Ihr Karsten Hadwich 